follow up in the 'abnormal' group [46.8 mm3 (37.1 - 64.6) vs. $44.6 \mathrm{~mm} 3(35.0$ - 57.7), $\mathrm{P}<0.001]$

Conclusion There was a differential response in plaque lipid reduction between normal and abnormal carotid arteries. Additionally, there was a significant degree of inconsistency in individual response to statin at the level of atherosclerotic plaque lipid content compared with LDL-c. Although LDL-c has a casual role in development of atherosclerosis, other lipid and non-lipid biomarkers have significant impact in determining reduction in atherosclerotic plaque lipid content. Therefore, LDL-c cannot be used, on its own, as a surrogate of plaque lipid reduction. Quantification of plaque lipid content may contribute to the identification of patients at greatest risk of atherosclerosis complications and to the targeting of this group with aggressive lipid lowering drugs.

Conflict of Interest None

\title{
6 CURRENT UTILITY OF FFRCT IN THE UNITED KINGDOM
}

Alex Asher*, George Thornton, Arvind Singhal, Ceri Davies, Andrew Wragg. Barts Health

10.1136/heartjnl-2019-BCS.6

Background In 2016 NICE produced Medical Technology Guidance approving the use of HeartFlow FFRCT for the investigation of patients presenting chest pain of new onset in the UK. The extent to which this technology has been adopted within the NHS in the UK is not yet known

Objectives Primarily we aimed to identify which centres in the UK are currently using CTCA with FFRCT technology and the total number of FFRCT scans compared to the total number of CTCAs in the 2016-17 financial year. Secondarily we aimed to identify which centres had capability for use of FFRCT technology in the coming years.

Methods A Freedom of Information request was submitted to all NHS trusts asking the following questions:

1. Does the trust have a 64-slice (or above) CT scanner?

2. Does the trust perform Coronary CT angiography?

3. How many Coronary CT Angiography scans were performed in the financial year 2016-2017?

4. Does your Trust have access to Coronary CT Angiography with non-invasive FFR analysis or HeartFlow FFRCT technology?

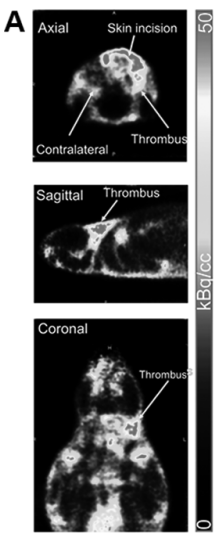

B

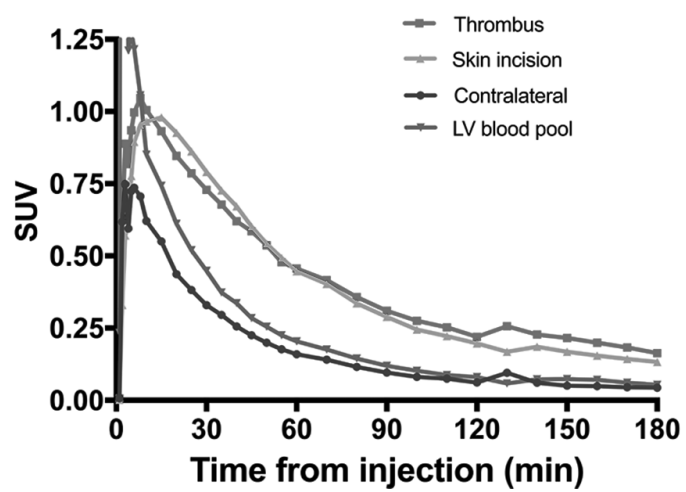

5. If so how many FFRCT scans have been performed in the financial year 2016-2017?

Results We achieved a 99\% response rate of all acute NHS trusts (159/161). $74 \%$ of all trusts reported providing a CTCA service. 70,928 CTCA scans were reported by these trusts. 12 trusts (8\%) reported having access to FFRCT technology although only half of these had performed FFRCT analysis in 2016-17. 11/12 trusts were located in England and $1 / 12$ in Scotland. Overall 210 FFRCT scans were performed in these 6 trusts. FFRCT analysis was used in just $0.3 \%$ of all the CTCAs in the UK in 2016-17. All trusts performing FFRCT scans performed at least 600 CTCAs per year. All FFRCT scans were requested in England in this period. A further $139 / 161$ trusts $(86 \%)$ have potential to set up a future FFRCT service based on this data.

Conclusion FFRCT technology remains limited to a handful of high volume centres. Only a small number of scans are being performed nationally but there is scope for significant expansion in years to come. There is marked geographical variation in availability of FFRCT currently.

Conflict of Interest Nil

\section{NON-INVASIVE IMAGING OF ACUTE THROMBOSIS; DEVELOPMENT OF A NOVEL FACTOR XIIIA RADIOTRACER}

${ }^{1}$ Jack Andrews*, ${ }^{1}$ Patrick Hadoke, ${ }^{2}$ Christophe Portal, ${ }^{1}$ Tashfeen Walton, ${ }^{1}$ Mark MacAskill, ${ }^{1}$ Christophe Lucatelli, ${ }^{3}$ Simon Wilson, ${ }^{2}$ lan Wilson, ${ }^{4}$ Gillian Macnaught, ${ }^{5}$ Marc Dweck, ${ }^{5}$ David Newby, ${ }^{5}$ Adriana Tavares. ${ }^{1}$ University of Edinburgh; ${ }^{2}$ Edinburgh Molecular Imaging Ltd; ${ }^{3}$ NHS Lothian; ${ }^{4}$ Edinburgh Imaging facility, Queens Medical Research Institute; ${ }^{5}$ British Heart Foundation Centre for Cardiovascular Science, University of Edinburgh

\subsection{6/heartjnl-2019-BCS.7}

Background Cardiovascular thrombosis is responsible for one quarter of all deaths annually worldwide. Current imaging methods focus on anatomical identification of thrombus but cannot determine thrombus age or activity. Molecular imaging techniques hold promise for identifying and quantifying active thrombosis in vivo. Our objective was to assess a novel optical and positron-emitting probe targeting Factor XIIIa (ENC2015) as biomarker of active thrombus formation.

Methods Optical and positron emitting ENC2015 probes were assessed ex vivo using blood drawn directly from human volunteers and passed through perfusion chambers containing denuded porcine aorta as a model of deep arterial injury.

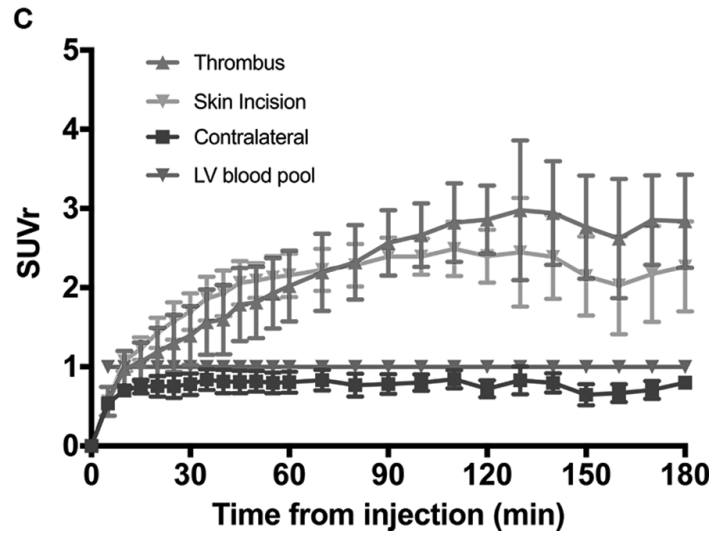

